\title{
Early mobilization improves functional outcomes in critically ill patients
}

\author{
Naishadh Brahmbhatt', Raghavan Murugan*1,2 and Eric B Milbrandt ${ }^{1,2}$ \\ University of Pittsburgh Department of Critical Care Medicine: Evidence-Based Medicine Journal Club, edited by Sachin Yende
}

\section{Expanded abstract Citation}

Schweickert WD, Pohlman MC, Pohlman AS, Nigos C, Pawlik AJ, Esbrook CL Spears L, Miller M, Franczyk M, Deprizio D, Schmidt GA, Bowman A, Barr R, McCalliste KE, Hall JB, Kress JP. Early physical and occupational therapy in mechanically ventilated, critically ill patients: a randomized controlled trial. Lancet 2009; 373(9678): 1874-1882. PubMed PMID: 19446324. This is available on www.pubmed.gov.

\section{Background}

Long-term complications of critical illness include intensive care unit (ICU)-acquired weakness and neuropsychiatric disease. Immobilization secondary to sedation might potentiate these problems.

\section{Methods}

Objective: To assess efficacy of combining daily interruption of sedation with physical and occupational therapy on functional outcomes in patients receiving mechanical ventilation in intensive care.

Design: Open label randomized clinical trial.

Setting: Study was conducted at two university hospitals on patients receiving sedation and mechanical ventilation. Subjects were those who received mechanical ventilation for $<72 \mathrm{hrs}$, were functionally independent prior to hospitalization, and were expected to continue for at least $24 \mathrm{hrs}$ after enrollment.

Subjects: 104 mechanically ventilated patients in the ICU. Intervention: Patients were randomized to receive either early exercise and mobilization (physical and occupational therapy) during periods of daily interruption of sedation (intervention; $n=49$ ) or daily interruption of

*Correspondence: muruganr@ccm.upmc.edu

642 Scaife Hall, 3550 Terrace Street, University of Pittsburgh, Pittsburgh, PA 15261, USA

Full list of author information is available at the end of the article sedation with therapy as ordered by the primary care team (control; $n=55$ ). Therapists who undertook patient assessments were blinded to treatment assignment.

Outcomes: The primary endpoint was the number of patients returning to independent functional status at hospital discharge defined as the ability to perform six activities of daily living and the ability to walk independently. Secondary endpoints included duration of delirium and ventilator-free days during the first 28 days of hospital stay.

\section{Results}

The return to independent functional status at hospital discharge occurred in 29 (59\%) patients in the intervention group compared with 19 (35\%) patients in the control group ( $\mathrm{p}=0.02$; odds ratio 2.7 [95\% CI 1.2-6.1]). Patients in the intervention group had shorter duration of delirium (median 2.0 days, IQR 0.0-6.0 vs 4.0 days, $2.0-8.0 ; \quad \mathrm{p}=0.02)$, and more ventilator-free days ( 23.5 days, $7.4-25.6$ vs 21.1 days, $0.0-23.8 ; \mathrm{p}=0.05$ ) during the 28-day follow-up period than did controls. There was one serious adverse event in 498 therapy sessions (desaturation less than 80\%). Discontinuation of therapy as a result of patient instability occurred in 19 (4\%) of all sessions, most commonly for perceived patient-ventilator asynchrony.

\section{Conclusions}

A strategy for whole-body rehabilitation consisting of interruption of sedation and physical and occupational therapy in the earliest days of critical illness was safe and well tolerated, and resulted in better functional outcomes at hospital discharge, a shorter duration of delirium, and more ventilator-free days compared with standard care.

\section{Commentary}

Technological advances in critical care have reduced mortality and resulted in the conversion of many lethal diseases to a syndrome of acute illness with long-term consequences $[1,2]$. Nevertheless, an important complication of exposure to critical illness is the associated 
increase in morbidity among survivors [3,4]. Interventions during critical illness, such as sedation, analgesia, or neuromuscular blockade with prolonged immobilization, contribute to neurocognitive impairment, physical debility, and ICU-acquired weakness, and result in significant long term morbidity and increased healthcare resource utilization $[3,5]$.

Healthy well nourished individuals show signs of skeletal muscle atrophy if immobilized for more than 72 hours [6], and older adults exhibit greater loss of muscle mass and strength compared to young adults with prolonged bed rest [7]. Observational studies show that early initiation of physical therapy (PT) in patients on mechanical ventilation is associated with more rapid return to ambulation, and improved functional independence $[8,9]$. Even repeated daily passive mobilization has been shown to prevent muscle atrophy in mechanically ventilated patients [10]. These findings suggest that preservation of muscle strength and architecture could improve functional outcome as both ICU-acquired paresis and handgrip strength have been independently associated with poor hospital outcomes [11].

In the current study, Schweickert and coworkers, [12] hypothesized that early initiation of PT and OT coupled with daily interruption of sedation would improve functional and neuropsychiatric outcomes. The study was conducted at two centers, none of which delivered routine PT within two weeks of initiation of mechanical ventilation. The study was conducted using a multidisciplinary approach and patient assessment for sedation and functional status was performed using widely validated tools and scales. Both groups received protocol driven mechanical ventilation, goal directed sedation with daily interruption, daily spontaneous breathing trials, and glycemic control. PT and OT were provided with a group of therapist different than those performing outcome assessments. Therapy sessions were safely provided with high compliance and very low adverse events in both groups throughout the study period. Patients in intervention group were more likely to achieve independent functional status, had fewer delirium and sedation days, and greater number of ventilator-free days than patients allocated to the control group. Intervention group patients also had higher activities of daily living as assessed using the Barthel scale. At hospital discharge, intervention patients ambulated farther without assistance and had increased tendency towards discharge to home. Importantly, the treatment given to the control group appeared to be a fair representation of routine ICU care in the community.

This was a well-done study addressing an important clinical problem. There are several important limitations that deserve consideration. First, it is important to note that only patients with independent functional status prior to illness were included in the study. It is unclear whether the findings of this study could be generalized to other critically ill populations, such as those with impaired premorbid functional status prior to critical illness. Whether early PT in such patients will result in preservation of baseline functional state is unknown. Second, the study population involved less severely ill patients (median APACHE II score in both arms 19.5 [Interquartile range, 14.5-23.5]) who were at lower risk for developing functional impairment. It remains to be seen whether early PT and OT will preserve function in more severely ill patients who are at a greater risk for functional impairment. Third, the unblinded nature of the study exposes the groups to potential biases caused by differential decisions of when to reinitiate sedation, the timing of extubation, and ICU and hospital discharge that may have accounted for differences in outcomes. Finally, despite reduction in days with delirium no information is provided about delirium and sedation scores at the time of assessment and diagnosis of delirium. Whether actual delirium was reduced, or instead patients were simply more awake due to implementation of early mobilization, PT, and daily sedation interruption is difficult to evaluate.

\section{Recommendation}

Schweickert and colleagues should be commended for showing us that the early administration of PT and OT in critical illness is safe, effective, and improves overall functional independence. It is unclear, however, whether these findings can be extrapolated across all spectrums of critically ill patients and a larger multicenter trial would be helpful in answering some of these questions. Even in the absence of such evidence, it would seem prudent do our best to minimize sedation and to initiate PT/OT as early as possible.

Competing interests

The authors declare that they have no competing interests.

\section{Author details}

'Department of Critical Care Medicine, University of Pittsburgh, Pittsburgh, PA, USA. ${ }^{2}$ The Clinical Research, Investigation, and Systems Modeling of Acute Illness (CRISMA) Center, University of Pittsburgh, Pittsburgh, PA, USA

Published: 24 September 2010

\section{References}

1. Ventilation with lower tidal volumes as compared with traditional tidal volumes for acute lung injury and the acute respiratory distress syndrome. The Acute Respiratory Distress Syndrome Network. N Engl J Med 2000, 342:1301-1308.

2. Kress JP, Pohlman AS, O'Connor MF, Hall JB: Daily interruption of sedative infusions in critically ill patients undergoing mechanical ventilation. NEngl J Med 2000, 342:1471-1477.

3. De Jonghe B, Lacherade JC, Durand MC, Sharshar T: Critical illness neuromuscular syndromes. Crit Care Clin 2007, 23:55-69.

4. Schweickert WD, Gehlbach BK, PohIman AS, Hall JB, Kress JP: Daily interruption of sedative infusions and complications of critical illness in mechanically ventilated patients. Crit Care Med 2004, 32:1272-1276. 
5. Ely EW, Shintani A, Truman B, Speroff T, Gordon SM, Harrell FE, Jr., Inouye SK, Bernard GR, Dittus RS: Delirium as a predictor of mortality in mechanically ventilated patients in the intensive care unit. JAMA 2004, 291:1753-1762.

6. Kortebein P, Ferrando A, Lombeida J, Wolfe R, Evans WJ: Effect of 10 days of bed rest on skeletal muscle in healthy older adults. JAMA 2007, 297:1772-1774.

7. lannuzzi-Sucich M, Prestwood KM, Kenny AM: Prevalence of sarcopenia and predictors of skeletal muscle mass in healthy, older men and women. $J$ Gerontol A Biol Sci Med Sci 2002, 57:M772-M777.

8. Bailey P, Thomsen GE, Spuhler VJ, Blair R, Jewkes J, Bezdjian L, Veale K, Rodriquez L, Hopkins RO: Early activity is feasible and safe in respiratory failure patients. Crit Care Med 2007, 35:139-145.

9. Morris PE, Goad A, Thompson C, Taylor K, Harry B, Passmore L, Ross A, Anderson L, Baker S, Sanchez M, Penley L, Howard A, Dixon L, Leach S, Small R, Hite RD, Haponik E: Early intensive care unit mobility therapy in the treatment of acute respiratory failure. Crit Care Med 2008, 36:2238-2243.

10. Griffiths RD, Palmer TE, Helliwell T, MacLennan P, MacMillan RR: Effect of passive stretching on the wasting of muscle in the critically ill. Nutrition 1995, 11:428-432.

11. Ali NA, O'Brien JM, Jr., Hoffmann SP, Phillips G, Garland A, Finley JC, Almoosa K, Hejal R, Wolf KM, Lemeshow S, Connors AF, Jr., Marsh CB: Acquired weakness, handgrip strength, and mortality in critically ill patients. $\mathrm{Am} J$ Respir Crit Care Med 2008, 178:261-268.

12. Schweickert WD, Pohlman MC, Pohlman AS, Nigos C, Pawlik AJ, Esbrook CL, Spears L, Miller M, Franczyk M, Deprizio D, Schmidt GA, Bowman A, Barr R, McCallister KE, Hall JB, Kress JP: Early physical and occupational therapy in mechanically ventilated, critically ill patients: a randomised controlled trial. Lancet 2009, 373:1874-1882.

doi:10.1186/cc9262

Cite this article as: Brahmbhatt N, et al:: Early mobilization improves functional outcomes in critically ill patients. Critical Care 2010, 14:321. 